SOI: $1.1 /$ TAS $\quad$ DOI: $10.15863 /$ TAS International Scientific Journal Theoretical \& Applied Science

\author{
p-ISSN: 2308-4944 (print) e-ISSN: 2409-0085 (online) \\ Year: 2017 Issue: $11 \quad$ Volume: 55 \\ Published: $28.11 .2017 \quad \underline{\text { http://T-Science.org }}$
}

Sergey Alexandrovich Mishchik Associate Professor, Candidate of Pedagogical Science, Academician of International Academy TAS, Assistant professor Department of Physics, State Maritime University Admiral Ushakov, Russia, sergei_mishik@mail.ru

SECTION 21. Pedagogy. Psychology. Innovation in Education

\title{
SYSTEMIC PROBLEMS CONSTANT MAGNETISM OF APPLIED PHYSICS MARITIME FLOT OF PEDAGOGOMETRIC ANALYSIS
}

Abstract: The basic principles of the system of problems constant magnetism in applied physics Navy pedagogometric analysis of the formation of mathematical models of learning activities about the nature of achieving the criteria of life, cycling, systemsness and phasing, which form a basic cell of the educational space, as well as prima nenie twelve pointed star Ertsgammy relatively presentation ertsgamming principle which determines the foundations pedagogometric through forming substantive methods of hyper-space professional life, psychological and educational activity theory, psycho-pedagogical system analysis and the theory of the formation of mental actions.

Key words: pedagogometric, vital activity, cyclicity, system, phase, star Erzgammy, constant magnetism, applied physics, marine fleet.

Language: Russian

Citation: Mishchik SA (2017) SYSTEMIC PROBLEMS CONSTANT MAGNETISM OF APPLIED PHYSICS MARITIME FLOT OF PEDAGOGOMETRIC ANALYSIS. ISJ Theoretical \& Applied Science, 11 (55): 219-227.

Soi: http://s-o-i.org/1.1/TAS-11-55-28 Doi: crossef https://dx.doi.org/10.15863/TAS.2017.11.55.28

\section{УДК 372.851}

\section{СИСТЕМНЫЕ ЗАДАЧИ ПОСТОЯННОГО МАГНЕТИЗМА ПРИКЛАДНОЙ ФИЗИКИ МОРСКОГО ФЛОТА ПЕДАГОГОМЕТРИЧЕСКОГО АНАЛИЗА}

Аннотация: Рассмотрены основные принципы построения системных задач постоянного магнетизма прикладной физики морского флота педагогометрического анализа при формировании математических моделей учебной деятельности относительно характера достижения критериев жизнедеятельности, иикличности, системности и этапности, которые образуют базисную ячейку образовательного пространства, а также применение двенадиати конечной звезды Эригаммы относительно представления принциипа эригаммности, который определит основы педагогометрики через формообразование предметными методами гиперпространства профессиональной жизнедеятельности, психолого-педагогической теории деятельности, психолого-педагогического системного анализа и теории формирования умственных действий.

Ключевые слова: педагогометрика, жизнедеятельность, ичикличность, системность, этапность, звезда Эригаммы, постоянный магнетизм, прикладная физика, морской флот.

\section{Introduction}

Образование системных задач постоянного магнетизма прикладной физики морского флота педагогометрического анализа направлено на решением общей задачи педагогометрики представление математических моделей учебной деятельности через базисный процесс методологии педагогометрического анализа, выражающего заданную структуру и форму жизнедеятельности, цикличности, системности и этапности. Предложенный педагогометрический анализ формирует базисную ячейку образовательного пространства, которая отражает принцип эрцгаммности через всеобщую структуру двенадцати конечной звезды Эрцгаммы. Установленная зависимость выражает основы педагогометрики через выделение предметных методов гиперпространства 
профессиональной

жизнедеятельности, психолого-педагогической теории деятельности, психолого-педагогического системного анализа и теории формирования умственных действий $[1,2,3]$.

Представленные основы подготовки инновационных специалистов широкопрофильных связываются с совершенствованием базы предметных прикладных профессиональных задач постоянного магнетизма прикладной физики морского флота педагогометрического анализа, через проективную профессиональную деятельность на морском флоте. Формирование математических моделей учебнопрофессиональной деятельности специалистов инновационного мышления ориентируется на: базисную звезду Эрцгаммы гиперпространства жизнедеятельности (Е1); базисный целостносистемный цикл жизнедеятельности (Е2); базисную звезду Эрцгаммы системного анализа (E3); базисное проявление двенадцати этапов и форм познавательного гиперпространства жизнедеятельности относительно образовательного процесса (E4) [4,5,6].

Особенности системных задач постоянного магнетизма прикладной физики морского флота педагогометрического анализа и проектная база предметных педагогометрических моделей эрцгаммного анализа образовательных объектов с признаком базисно-нормативной эрцгаммности, определяют их обобщённые структуры. В результате формируется собственная функция психолого-математического представления профессионально-значимых объектов системных задач постоянного магнетизма прикладной физики морского флота педагогометрического анализа через объединение признаков смыслообразования учебно-профессионального действия, его принятия, ориентировочноисполнительно-контрольных признаков и прогноза совершенствования анализа объектов педагогометрческого содержания [7,8,9].

\section{Materials and Methods}

Системные задачи постоянного магнетизма прикладной физики морского флота предопределяют целостно-системное моделирование основных элементов транспортных технических объектов. Это приводит к формированию сознательной ориентации на единство базисных характеристик предметных и исполнительных условий относительно предмета содержания и способа его реализации через представление базисной ячейки образовательного пространства, которая отражает принцип эрцгаммности адекватного структуре двенадцати конечной звезды Эрцгаммы.
Рассматриваются: напряженность магнитного поля в судовой антенне; базисная напряженность магнитного поля в центре кругового проволочного витка-датчика судовой системы электронного контроля; напряженности магнитного поля, вызванного токами судовой электрической системы; определение число слоев обмотки соленоида судовой дроссельной катушки системы автоматического контроля на морском флоте [10, 11,12].

В процессе решения системных задач постоянного магнетизма прикладной физики морского флота необходимо применять основные положения теории деятельности, системного анализа и теории формирования интеллекта через построение математических моделей учебнопрофессиональной активности отражающей структуру: базисной звездой Эрцгаммы гиперпространства жизнедеятельности (E1); базисного целостно-системного циклом жизнедеятельности (E2); базисной звездой Эрцгаммы системного анализа (Е3); базисного проявления двенадцати этапов и форм познавательного гиперпространства жизнедеятельности относительно образовательного процесса (E4).

Системный анализ предполагает выполнение последовательности системных аналитических действий: выделить объект анализа - задачу постоянного магнетизма прикладной физики морского флота (ЗПМПФМФ) как систему; установить порождающую среду ЗПМПФМФ; определить уровни анализа ЗПМПФМФ; представить целостные свойства ЗПМПФМФ относительно пространственных, и временных характеристик и их комбинаций; выделить структуру уровня анализа ЗПМПФМФ; установить структурные элементы уровня анализа ЗПМПФМФ; определить системообразующие связи данного уровня анализа ЗПМПФМФ; представить межуровневые связи анализа ЗПМПФМФ; выделить форму организации ЗПМПФМФ; установить системные свойства и поведение ЗПМПФМФ.

\section{Задача 1}

В судовой горизонтальной антенне в виде длинного провода в процессе передачи возникает ток $\boldsymbol{I}=\mathbf{5}$ Aмnер. Определить напряженность $\boldsymbol{H}$ магнитного поля в точках, находящихся на расстоянии $\boldsymbol{a}=\mathbf{2} \boldsymbol{M}$ от судовой горизонтальной антенны.

Ответ: $H=39,8 \mathrm{~A} / \mathrm{M}$.

\section{Задача 2}

Судовая система электронного контроля имеет круговой проволочный виток-датчик радиусом $\boldsymbol{R}=\boldsymbol{l} \boldsymbol{c m}$, по которому следует ток $\boldsymbol{I = \boldsymbol { I }}$ Aмnep. Определить базисную напряженность $\boldsymbol{H}$ 


\begin{tabular}{|c|c|c|c|c|c|c|}
\hline Impact Factor: & $\begin{array}{l}\text { ISRA (India) } \\
\text { ISI (Dubai, UAE } \\
\text { GIF (Australia) } \\
\text { JIF }\end{array}$ & $\begin{array}{r}=1.344 \\
=0.829 \\
=0.564 \\
=1.500\end{array}$ & $\begin{array}{l}\text { SIS (USA) } \\
\text { PИНЦ (Russia) } \\
\text { ESJI (KZ) } \\
\text { SJIF (Morocco) }\end{array}$ & $\begin{array}{l}=0.912 \\
=0.207 \\
=\mathbf{3 . 8 6 0} \\
=\mathbf{2 . 0 3 1}\end{array}$ & $\begin{array}{l}\text { ICV (Poland) } \\
\text { PIF (India) } \\
\text { IBI (India) }\end{array}$ & $\begin{array}{l}=6.630 \\
=1.940 \\
=4.260\end{array}$ \\
\hline
\end{tabular}

магнитного поля в центре кругового проволочного витка-датчика судовой системы электронного контроля.

Ответ: $H=50 \mathrm{~A} / \mathrm{M}$.

Задача 3

На рисунке изображено сечение судовой электрической системы в виде двух прямолинейных проводников с токами $\boldsymbol{I}_{\boldsymbol{1}}$ и $\boldsymbol{I}_{2}$. Расстояние между проводниками судовой электрической системы $\boldsymbol{A B}=10 \mathrm{~cm}$, токи $\boldsymbol{I}_{I}=20 \mathrm{~A}$ и $I_{2}=30 \quad \boldsymbol{A}$. Определить напряженности $\boldsymbol{H}$ магнитного поля, вызванного токами судовой электрической системы $\boldsymbol{I}_{1}$ и $\boldsymbol{I}_{2}$ в точках $\boldsymbol{M}_{1}, \boldsymbol{M}_{2} \boldsymbol{u}$ M. Расстояния $M_{1} A=2$ c.м, $A M_{2}=4$ см и $B M_{3}=3$ c.M.

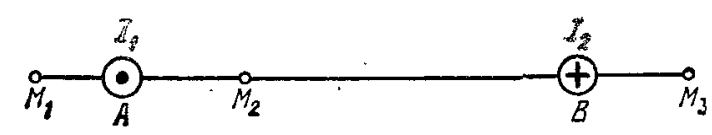

Рисунок 1 - Сечение судовой электрической системы.

Ответ: $H_{1}=120 \mathrm{~A} / \mathrm{M} ; \quad \mathrm{H}_{2}=159 \mathrm{~A} / \mathrm{m}$;

Задача 4

На рисунке изображено сечение судовой электрической системы в виде двух прямолинейных проводников с токами $\boldsymbol{I}_{1}$ и $\boldsymbol{I}_{2}$.
Расстояние между проводниками судовой электрической системы $\boldsymbol{A B}=10 \mathrm{~cm}$, токи $\boldsymbol{I}_{1}=20 \boldsymbol{A}$ и $\boldsymbol{I}_{2}=\mathbf{3 0} \boldsymbol{A}$. Определить напряженности $\boldsymbol{H}$ магнитного поля, вызванного токами судовой электрической системы $\boldsymbol{I}_{\boldsymbol{1}}$ и $\boldsymbol{I}_{2}$ в точках $\boldsymbol{M}_{\boldsymbol{1}}, \boldsymbol{M}_{2} \boldsymbol{u}$ M. Расстояния $M_{1} A=2$ с.м, $A M_{2}=4$ см и $B M_{3}=3$ c.M.

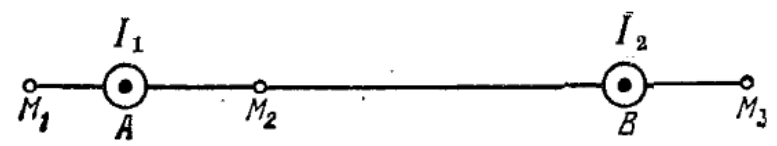

Рисунок 2 - Сечение судовой электрической системы.

Ответ: $H_{1}=199 \mathrm{~A} / \mathrm{M} ; \mathrm{H}_{2}=0 \mathrm{~A} / \mathrm{M} ; \mathrm{H}_{3}=183 \mathrm{~A} / \mathrm{M}$.

Задача 5

На рисунке изображено сечение судовой электрической системы в виде трёх прямолинейных проводников с токами $\boldsymbol{I}_{1}, \boldsymbol{I}_{2}$ и $\boldsymbol{I}_{3}$. Расстояния $\mathrm{AB}=\mathrm{BC}=5 \mathrm{~cm}$, токи $\boldsymbol{I}_{\boldsymbol{I}}=\boldsymbol{I}_{2}=\boldsymbol{I}$ и $\boldsymbol{I}_{3}=2 \boldsymbol{I}$. Определить точку на прямой $\mathrm{AC}$, в которой напряженность магнитного поля, вызванного токами $\boldsymbol{I}_{1}, \boldsymbol{I}_{2}$ и $\boldsymbol{I}_{3}$, равна нулю.

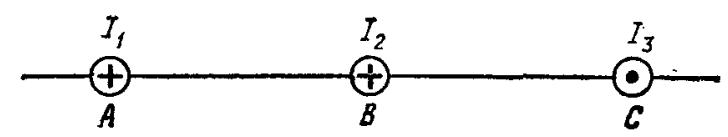

Рисунок 3 - Сечение судовой электрической системы.

Ответ: между точками $I_{1}$ и $I_{2} \quad$ на расстоянии а $=3,3$ см от точки $A$.

Задача 6

На рисунке изображено сечение судовой электрической системы в виде трёх прямолинейных проводников с токами $\boldsymbol{I}_{1}, \boldsymbol{I}_{2}$ и $\boldsymbol{I}_{3}$. Расстояния $\mathrm{AB}=\mathrm{BC}=5 \mathrm{~cm}$, токи $\boldsymbol{I}_{\boldsymbol{I}}=\boldsymbol{I}_{2}=\boldsymbol{I}$ и $\boldsymbol{I}_{3}=2 \boldsymbol{I}$. Определить точку на прямой $\mathrm{AC}$, в которой напряженность магнитного поля, вызванного токами $\boldsymbol{I}_{1}, \boldsymbol{I}_{2}$ и $\boldsymbol{I}_{3}$, равна нулю.

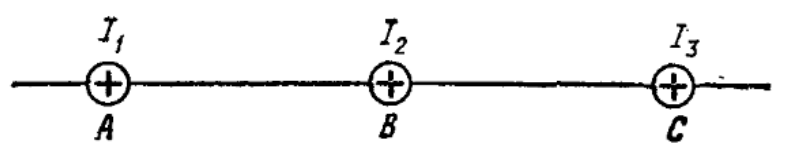

Рисунок 4 - Сечение судовой электрической системы.

ISPC Technological development, 


\begin{tabular}{|c|c|c|c|c|c|c|}
\hline Impact Factor: & $\begin{array}{l}\text { ISRA (India) } \\
\text { ISI (Dubai, UAF } \\
\text { GIF (Australia) } \\
\text { JIF }\end{array}$ & $\begin{array}{l}=1.344 \\
=0.829 \\
=0.564 \\
=1.500\end{array}$ & $\begin{array}{l}\text { SIS (USA) } \\
\text { PИНЦ (Russia) } \\
\text { ESJI (KZ) } \\
\text { SJIF (Morocco) }\end{array}$ & $\begin{array}{l}=0.912 \\
=0.207 \\
=\mathbf{3 . 8 6 0} \\
=\mathbf{2 . 0 3 1}\end{array}$ & $\begin{array}{l}\text { ICV (Poland) } \\
\text { PIF (India) } \\
\text { IBI (India) }\end{array}$ & $\begin{array}{l}=6.630 \\
=1.940 \\
=4.260\end{array}$ \\
\hline
\end{tabular}

Ответ: правее точки $А$ на расстояниях $a_{1}$ $=1,8 \mathrm{~cm}$ и $a_{2}=6,96 \mathrm{~cm} \mathrm{om} \mathrm{нее.}$

\section{Задача 7}

Два прямолинейных длинных проводника судовой электрической системы расположены перпендикулярно друг к другу и находятся в одной плоскости. Определить напряженности $\boldsymbol{H}_{\boldsymbol{I}}$ и $\boldsymbol{H}_{2}$ магнитного поля в точках $\boldsymbol{M}_{1}$ и $\boldsymbol{M}_{2}$ судовой электрической системы, если токи $\boldsymbol{I}_{1}=2 \boldsymbol{A}$ и $\boldsymbol{I}_{2}=3$ $A$. Расстояния $A M_{1}=A M_{2}=1 c \cdot$ и $\boldsymbol{B M}_{1}=C M_{2}=2 \mathrm{~cm}$.

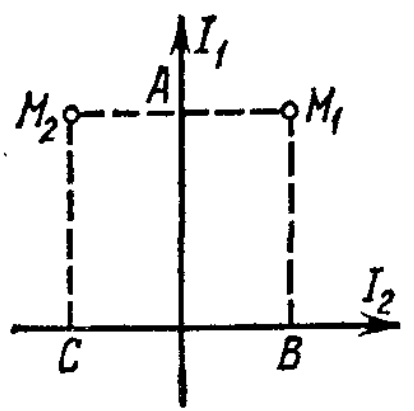

Рисунок 5 - Сечение судовой электрической системы.

Ответ: $H_{1}=8 \mathrm{~A} / \mathrm{M} ; \mathrm{H}_{2}=55,8 \mathrm{~A} / \mathrm{M}$.

\section{Задача 8}

Два прямолинейных бесконечно длинных проводника судовой электрической системы расположены перпендикулярно друг к другу и находятся во взаимно перпендикулярных плоскостях. Определить напряженности $\boldsymbol{H}_{\boldsymbol{I}}$ и $\boldsymbol{H}_{2}$ магнитного поля судовой электрической системы в точках $\boldsymbol{M}_{1}$ и $\boldsymbol{M}_{2}$, если токи $\boldsymbol{I}_{1}=2 \boldsymbol{A}$ и $\boldsymbol{I}_{2}=3 \boldsymbol{A}$. Расстояния $A M_{1}=A M_{2}=l c m$ и $A B=2 \mathrm{~cm}$.

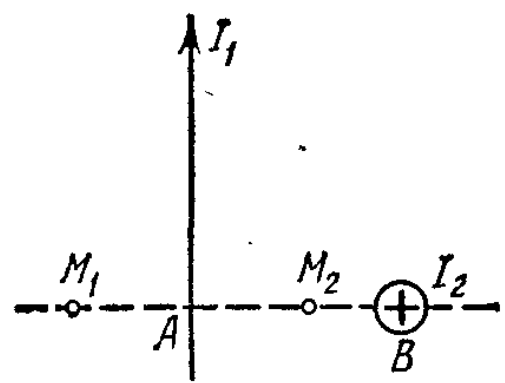

Рисунок 6 - Сечение судовой электрической системы.

Ответ: $H_{1}=35,6 \mathrm{~A} / \mathrm{M} ; \mathrm{H}_{2}=57,4 \mathrm{~A} / \mathrm{M}$.

\section{Задача 9}

Два прямолинейных длинных проводника судовой электрической системы расположены параллельно на расстоянии $\boldsymbol{d}=\mathbf{1 0} \boldsymbol{c m}$ друг от друга. По проводникам распространяются токи $\boldsymbol{I}_{\boldsymbol{I}}$ $=\boldsymbol{I}_{2}=5 \boldsymbol{A}$ в противоположных направлениях. Определить модуль и направление напряженности $\boldsymbol{H}$ магнитного поля в точке судовой электрической системы, находящейся на расстоянии $\boldsymbol{a}=10 \mathrm{~cm}$ от каждого проводника.

Ответ: $\boldsymbol{H}=8 \mathrm{~A} / \mathrm{s}$. Напряженность магнитного поля направлена перпендикулярно к плоскости, проходящей через оба провода.

\section{Задача 10}

В судовой электрической системе определить напряженность $\boldsymbol{H}$ магнитного поля, создаваемого отрезком $\boldsymbol{A B}$ прямолинейного проводника с током судовой электрической системы, в точке $\boldsymbol{C}$, расположенной на перпендикуляре к середине этого отрезка на расстоянии $\boldsymbol{a = 5} \boldsymbol{c m}$. По проводнику судовой электрической системы следует сила тока $\boldsymbol{I}=\mathbf{2 0}$ $\boldsymbol{A}$. Отрезок $\boldsymbol{A B}$ проводника виден из точки $\boldsymbol{C}$ под углом $60^{\circ}$.

Ответ: $H=31,8 \mathrm{~A} / \mathrm{M}$.

\section{Задача 11}

В судовой электрической системе определить напряженность $\boldsymbol{H}$ магнитного поля, создаваемого отрезком $\boldsymbol{A B}$ прямолинейного проводника с током судовой электрической 
системы, в точке $\boldsymbol{C}$, расположенной от этого отрезка на расстоянии $\boldsymbol{a}=\mathbf{5} \boldsymbol{c m}$. По проводнику судовой электрической системы следует сила тока $\boldsymbol{I}=\mathbf{3 0} \boldsymbol{A}$. Отрезок $\boldsymbol{A B}$ проводника виден из точки $\boldsymbol{C}$ под углом $90^{\circ}$.

Ответ: $H=56,5 \mathrm{~A} / \mathrm{M}$.

\section{Задача 12}

В судовой электрической системе отрезок прямолинейного проводника с током имеет длину $\boldsymbol{\ell}=30 \boldsymbol{c m}$. При каком предельном расстоянии $\boldsymbol{a}$ от него для точек судовой электрической системы, лежащих на перпендикуляре к его середине, магнитное поле можно рассматривать как поле бесконечно длинного прямолинейного тока? Ошибка при таком допущении не должна превышать $\boldsymbol{\varepsilon}=\mathbf{5 \%}$.

Ответ: $a<5 \mathrm{~cm}$.

\section{Задача 13}

В судовой электрической системе в точке $\boldsymbol{C}$, расположенной на расстоянии $\boldsymbol{a}=\mathbf{5} \boldsymbol{c m}$ от бесконечно длинного прямолинейного проводника с током, напряженность магнитного поля $\boldsymbol{H}=400 \boldsymbol{A} / \boldsymbol{M}$. При какой предельной длине $\boldsymbol{\ell}$ проводника судовой электрической системы это значение напряженности будет верным с точностью до $\boldsymbol{\varepsilon}=\mathbf{2 \%}$ ? Определить напряженность $\boldsymbol{H}$ магнитного поля в точке $\boldsymbol{C}$ судовой электрической системы, если проводник с током имеет длину $\boldsymbol{\ell}=\mathbf{2 0} \boldsymbol{c \boldsymbol { m }}$ и точка $\boldsymbol{C}$ расположена на перпендикуляре к середине этого проводника судовой электрической системы.

Ответ: $\ell>0,245 \mathrm{~m} ; \quad H=358 \mathrm{~A} / \mathrm{M}$.

\section{Задача 14}

В судовой электрической системе сила тока $\boldsymbol{I}$ $=20 \boldsymbol{A}$ существует в длинном проводнике, согнутым под прямым углом. Определить напряженность $\boldsymbol{H}$ магнитного поля в точке судовой электрической системы, находящейся на биссектрисе этого угла и отстоящей от вершины угла судовой электрической системы на расстоянии $\boldsymbol{a}=10 \mathrm{~cm}$.

Ответ: $H=77,3 \mathrm{~A} / \mathrm{M}$.

\section{Задача 15}

В судовой электрической системе сила тока $\boldsymbol{I}$ $=20 \boldsymbol{A}$, существует в кольце из медной проволоки сечением $\boldsymbol{S}=\boldsymbol{l , 0} \boldsymbol{м m}^{2}$, создает в центре кольца напряженность магнитного поля $\boldsymbol{H}=\mathbf{1 7 8} \boldsymbol{A} / \boldsymbol{M}$. Определить разность потенциалов $\boldsymbol{U}$ на концах проволоки судовой электрической системы, образующей кольцо. Удельное электрическое сопротивление медного проводника $\boldsymbol{\rho}=\mathbf{0 , 0 1 7}$ $\boldsymbol{м \kappa} \boldsymbol{O} \cdot \boldsymbol{M}$.

Ответ: $U=0,12 \mathrm{~B}$.
В судовой электрической системе определить напряженность $\boldsymbol{H}$ магнитного поля на оси кругового контура на расстоянии $\boldsymbol{a}=\mathbf{3} \boldsymbol{c \boldsymbol { M }}$ от его плоскости судовой электрической системы. Радиус контура судовой электрической системы $\boldsymbol{R}=4 \boldsymbol{c m}$, сила тока в контуре судовой электрической системы $\boldsymbol{I}=\mathbf{2} \boldsymbol{A}$.

Ответ: $H=12,7 \mathrm{~A} / \mathrm{M}$.

\section{Задача 17}

В судовой электрической системе напряженность магнитного поля в центре кругового витка $H_{o}=63,7 \mathrm{~A} / \mathrm{M}=1$ Эрстэд. Радиус витка $\boldsymbol{R}=1 \boldsymbol{1} \boldsymbol{c \boldsymbol { M }}$. Найти напряженность $\boldsymbol{H}$ магнитного поля на оси витка судовой электрической системы на расстоянии $\boldsymbol{a}=10 \mathrm{~cm}$ от его плоскости.

Ответ: $H=25,7 \mathrm{~A} / \mathrm{M}$.

\section{Задача 18}

В судовой электрической системе два круговых витка радиусом $\boldsymbol{R}=4 \boldsymbol{c m}$ расположены в параллельных плоскостях на расстоянии $\boldsymbol{d}=\mathbf{1 0}$ $\boldsymbol{c \boldsymbol { M }}$ друг от друга. По виткам следуют токи $\boldsymbol{I}_{\boldsymbol{1}}=\boldsymbol{I}_{2}$ $=2 \boldsymbol{A}$. Определить напряженность $\boldsymbol{H}$ магнитного поля на оси витков в точке судовой электрической системы, находящейся на равном расстоянии от них. Задачу решить, когда: а) токи в витках следуют в одном направлении; б) токи в витках следуют в противоположных направлениях

Ответ: а) $H=12,2 \mathrm{~A} / \mathrm{s}$; б) $H=0$.

\section{Задача 19}

В судовой электрической системе два круговых витка радиусом $\boldsymbol{R}=4 \boldsymbol{c m}$ расположены в параллельных плоскостях на расстоянии $\boldsymbol{d}=\mathbf{5} \boldsymbol{c m}$ друг от друга. По виткам следуют текут токи $\boldsymbol{I}_{\boldsymbol{I}}=$ $\boldsymbol{I}_{2}=\boldsymbol{4} \boldsymbol{A}$. Определить напряженность $\boldsymbol{H}$ магнитного поля в центре одного из витков судовой электрической системы. Задачу решить, когда: а) токи в витках судовой электрической системы следуют в одном направлении; б) токи в витках судовой электрической системы следуют в противоположных направлениях.

Ответ: а) $\mathrm{H}=62,2 \mathrm{~A} / \mathrm{M}$; б) $\mathrm{H}=38,2 \mathrm{~A} / \mathrm{M}$.

\section{Задача 20}

В судовой электрической системе два круговых витка расположены в двух взаимно перпендикулярных плоскостях так, что центры этих витков совпадают. Радиус каждого витка судовой электрической системы $\boldsymbol{R}=\mathbf{2} \boldsymbol{c m}$, токи в витках $\boldsymbol{I}_{\boldsymbol{1}}=\boldsymbol{I}_{2}=\mathbf{5} \boldsymbol{A}$. Определить напряженность $\boldsymbol{H}$ магнитного поля в центре этих витков судовой электрической системы.

Ответ: $\boldsymbol{H}=177 \mathrm{~A} / \mathrm{M}$. 
В судовой электрической системе из проволоки длиной $\boldsymbol{\ell}=\boldsymbol{1} \boldsymbol{м}$ сделана квадратная рамка. По рамке следуют ток $\boldsymbol{I}=\boldsymbol{5} \boldsymbol{A}$. Найти напряженность $\boldsymbol{H}$ магнитного поля в центре рамки судовой электрической системы.

Ответ: $H=35,8 \mathrm{~A} / \mathrm{M}$.

\section{Задача 22}

В судовой электрической системе в центре кругового проволочного индукционного датчикавитка создается магнитное поле напряженностью $\boldsymbol{H}$ при разности потенциалов $\boldsymbol{U}_{\boldsymbol{I}}$ на концах витка. Какую надо приложить разность потенциалов $\boldsymbol{U}_{2}$, чтобы в судовой электрической системе получить такую же напряженность магнитного поля в центре индукционного датчика-витка вдвое большего радиуса, сделанного из той же проволоки ?

Ответ: $U_{2}=4 U_{1}$.

\section{Задача 23}

В судовой электрической системе по проволочной рамке индукционного датчикавитка, имеющей форму правильного шестиугольника, следует сила тока $\boldsymbol{I}=\mathbf{5 A}$. При этом в центре судовой рамки индукционного датчика-витка образуется магнитное поле напряженностью $\boldsymbol{H}=\mathbf{3 3} \mathbf{A} / \mathbf{M}$. Найти длину $\boldsymbol{\ell}$ проволоки судовой рамки индукционного датчика-витка.

Ответ: $\ell=0,2$ м.

\section{Задача 24}

Длинный провод судовой электрической линии образует круговой виток индукционного датчика, касательный к проводу главной магистрали. По проводу судовой электрической линии следует сила тока $\boldsymbol{I}=\boldsymbol{5} \boldsymbol{A}$. Определить радиус $\boldsymbol{R}$ витка индукционного датчика, касательного к судовому проводу главной магистрали, если напряженность магнитного поля в центре витка $\boldsymbol{H}=\mathbf{4 1} \boldsymbol{A} / \boldsymbol{M}$.

Ответ: $R=8$ c. .

\section{Задача 25}

Судовая дроссельная катушка системы автоматического контроля имеет длину $\boldsymbol{\ell}=\mathbf{0 , 3} \boldsymbol{M}$ и $N=1000$ витков провода. Найти напряженность $\boldsymbol{H}$ магнитного поля внутри судовой дроссельной катушки, если по катушке следует сила тока $\boldsymbol{I}=$ $\boldsymbol{2 A}$. Диаметр судовой дроссельной катушки считать малым по сравнению с ее длиной.

Ответ: $H=1,25 \kappa \mathrm{A} / \mathrm{M}$.

\section{Задача 26}

Судовая дроссельная катушка системы автоматического контроля представляет собой соленоид из проволоки диаметром $\boldsymbol{d = 1} \boldsymbol{\boldsymbol { M }}$. Напряженность магнитного поля внутри соленоида составляет $\boldsymbol{H}=\mathbf{2 4} \boldsymbol{\kappa} \boldsymbol{A} / \boldsymbol{M}$. По судовой дроссельной катушке системы автоматического контроля можно пропускать предельную силу тока $\boldsymbol{I}=\boldsymbol{6} \boldsymbol{A}$. Определить число слоев обмотки соленоида судовой дроссельной катушки системы автоматического контроля, если витки наматывать плотно друг к другу. Диаметр соленоида считать малым по сравнению с его длиной.

\section{Ответ: из 4 слоев.}

\section{Задача 27}

В судовом дроссельном соленоиде системы автоматического контроля требуется получить напряженность магнитного поля $\boldsymbol{H}=\boldsymbol{1} \boldsymbol{\kappa A} / \boldsymbol{M}$. Судовой дроссельный соленоид системы автоматического контроля имеет длину $\boldsymbol{\ell}=\mathbf{2 0} \boldsymbol{c m}$ и диаметр $\boldsymbol{D}=\mathbf{5} \boldsymbol{c m}$. Определить число ампервитков IN судового дроссельного соленоида и разность потенциалов $\boldsymbol{U}$, которую надо приложить к концам обмотки из медной проволоки диаметром $\boldsymbol{d}=\mathbf{0 , 5} \boldsymbol{M м}$, чтобы получить заданную напряженность магнитного поля. Считать поле в судовом дроссельном соленоиде однородным.

Ответ: $I N=200 A \cdot \boldsymbol{A} ; U=2,7 \mathrm{~B}$.

\section{Задача 28}

В судовом дросселе системы автоматического контроля замкнутый железный сердечник длиной $\boldsymbol{\ell}=\mathbf{5 0} \boldsymbol{c m}$ имеет обмотку из $N=1000$ витков провода. По обмотке следует сила тока $\boldsymbol{I}_{\boldsymbol{1}}=\boldsymbol{1} \boldsymbol{A}$. Определить силу тока $\boldsymbol{I}_{2}$ в судовом дросселе системы автоматического контроля, чтобы при удалении сердечника индукция судового дросселя не изменилась.

Ответ: $I_{2}=620 \mathrm{~A}$.

\section{Задача 29}

Судовой дроссель системы автоматического контроля состоит из железного сердечника длиной $\boldsymbol{\ell}_{\boldsymbol{1}}=\mathbf{5 0 , 2} \boldsymbol{c \boldsymbol { M }}$ с воздушным зазором длиной $\boldsymbol{\ell}_{2}=\mathbf{0 , 1} \boldsymbol{c м}$ и имеет обмотку из $\mathrm{N}=\mathbf{2 0}$ витков провода. Определить силу тока $\boldsymbol{I}$ в судовом дросселе системы автоматического контроля, чтобы в зазоре дросселя получить индукцию магнитного поля $\boldsymbol{B}_{2}=1,2 \mathrm{~T} \boldsymbol{л}$.

Ответ: $I=60 \mathrm{~A}$.

\section{Задача 30}

Определить индукцию магнитного поля судового магнитного индикатора, если площадь контакта с металлической поверхностью равна $\boldsymbol{S}=22 \boldsymbol{c m}^{2}$, а сила отрыва судового магнитного индикатора от контролируемой судовой поверхности равна $\boldsymbol{F}=\mathbf{9 3 H}$. Массу судового магнитного индикатора в момент отрыва от контролируемой судовой поверхности не учитывать.

Ответ: $B=0,3258 T_{\pi}$. 


\section{Conclusion}

Особенности формирования и развития инновационного широкопрофильного профессионального мышления связывается с организацией всестороннего развития педагогометрической эрцгаммности. Представленные системные задачи постоянного магнетизма прикладной физики морского флота педагогометрического анализа выделяют основные направления развития и совершенствования базы прикладных предметных педагогометрических моделей образовательных объектов относительно педагогометрического математического моделирования учебного процесса. Развиваемая образовательная деятельность связывается с процессами совершенствования программируемых математических моделей учебной активности относительно характера достижения критериев жизнедеятельности, цикличности, системности и этапности.

Представленные содержательные задачи педагогометрического анализа ориентируются на выработку целостно-системного цикла жизнедеятельности, отражающего последовательность выполняемых фазовых состояний реализации данных учебного процесса, характеризующего исследуемые образовательные явления и представленных во времени в форме различных математических моделей.

Формирование целостно-системного цикла жизнедеятельности есть многоэтапный процесс. Преобразование внешнего образа мира во внутренний происходит в результате постепенной различно функциональной деятельности, которая отражает базисные рефлекторно-физиологические этапы процесса интериоризации относительно общего процесса познания. Педагогометрический анализ данных процессов позволит проводит плановое моделирование условиями подготовки широкопрофильных специалистов и управление формированием профессиональных качеств личности.

Определение в качестве базисного этапа формирования целостно-системной внутренней деятельности процесса ориентации, отражает предметный смысл всей психологопедагогической науки относительно подготовки современных целостно-системных широкопрофильных специалистов, имеющих высший уровень целостно-системной ориентировки в социально-экономических, технических и естественных системах.

Реализация поставленных педагогометрических задач происходит через последовательность педагогометрических действий. Психологопедагогический системный анализ занимает базисную позицию в формировании целостносистемной личности и её профессионального образа - специалиста широкого профиля. Рассматривая весь педагогометрический процесс вместе со всеми двенадцатью дей-ствиями педагогометрического системного анализа первое базисное действие «Выделить объект изучения как систему» обеспечивает абсолютную рефлексию относительно внутренней и внешней среды всей совокупности действий системного анализа и является своеобразной «Азбукой Жизни педагогометрического анализа» относительно всех форм активности субъекта образовательного пространства.

Первое действие педагогометрического системного анализа - «Выделить объект изучения как систему» выступает в двух основных задачах: 1) определяет характер учебнопрофессиональной деятельности; 2) задаёт схему оперативного мышления при анализе выделенного образа образовательно-деятельного пространства. Эти задачи определяют две основные технологии познавательно-творческого освоения мира: 1) многоуровневый, целостносистемный и циклический характер жизнедеятельности; 2) поэтапный метод формирования интеллекта в широком смысле относительно общего способа познавательного процесса.

Это определяет общий характер существования всей совокупности действий педагогометрического системного анализа, которые задают особый смысл всей технологии учебно-профессиональной деятельности. В первую очередь весь педагогометрический анализ выстраивает всю систему не только учебного предмета прикладной физики морского флота, но и метода организации его усвоения. Именно во введении в данный предмет прикладной физики морского флота раскрывается общая структура метода педагогометрического системного анализа предмета, его базисность, фундаментальность и ши-рокопрофильность учебно-профессиональных задач на примере постоянного магнетизма. При этом выделяются педагогометрические критерии уровня целостносистемного усвоения пред-мета. Переходя к основной части освоения учебной программы, именно первое действие системного анализа «Выделить объект изучения как систему» задаёт всю смысловую нагрузку раскрытия содержания всего предмета изучения с целью очерчивания его всеобщей, инвариантной структуры. высшего смысла акмеологического образа.

Последующий процесс освоения учебного предмета прикладной физики морского флота сводится к рассмотрению многовариантного 
образа смысла освоения учебной деятельности и презентации профессиональных умений. Множество формирующихся компетенций зарождаются именно с первого действия «Выделить объект изучения как систему». Вся система компетенций отражается «полный жизненный цикл» многовариантных учебнопрофессиональных умений, направленных на формирование двигательного навыка заданной скорости выполнения. Ограничение свободы познавательного действия или профильного исполнения выражает характер мастерства субъектов образовательного процесса профессионального содержания.

Заключительный этап разработки учебного предмета прикладной физики морского флота ориентируется на выделение контрастных учебных объектов, которые регулируют уровень учебно-профессионального знания. Процесс решения этих задач невозможен вне освоения первого действия системного анализа «Выделить объект изучения как систему». При этом выдвигается глобальная проблема формирования творчества - его нормативной основы, когда всякое новое и прогноз развития определяется в русле целостно-системных циклических концепций, направленных на формирование широкопрофильного мышления и нового типа личности.

Процесс освоения программы прикладной физики морского флота происходит через новую типологию учебных задач, которая задаётся основным из двенадцати действий педагогометрического системного анализа. Поэтому первый тип учебных задач определяется структурой первого действия педагогометрического системного анализа «Выделить объект изучения как систему». В каждой новой фазе познавательного цикла первое действие набрасывает ориентировочные контуры системного образа, когда с минимальным приближением определяются двенадцать основных результатов, операций системного представления: определяются общие контуры как педагогометрическая система; намечается порождающая среда; предформируются общие целостные свойства предмета прикладной физики морского флота; намечаются уровни анализа системы; приблизительно устанавливается структура каждого уровня; определяются общие границы каждого структурного элемента уровня; намечаются системообразующие связи системы; предформируются межуровневые связи системы; приблизительного устанавливается форма существования системы и каждого уровня; определяются общие контуры системных свойств предмета анализа по характеристикам сложности, разнообразия и упорядоченности; намечается характер поведения системы по статическим и динамическим параметрам и переходным процессам от статической статики до динамической динамики; предформируюся прогнозы развития системы с выделением трендовых закономерностей $[13,14,15]$.

При формировании структуры целостносистемного учебно-профессионального широкопрофильного цикла действие системного анализа

«Выделить объект изучения как систему» применяется не только при анализе предметных условий, но и самих форм деятельности: всеобеей, технологической, контрольной, ритуальной, восходящей, развивающей и смыслового скачка. Поэтому при выполнении традиционных форм учебного процесса: лекции; практические и лабораторные занятия - сама модульная структура познавательного действия в прикладной физике морского флота, должна отражать системную педагогометрическую структуру.

Применение первого действия системного анализа «Выделить объект изучения как систему» в процессе формирования основных форм знания: мотивационной, ориентационной, материальной, внешнеречевой, вербально-знаковой и их дополнительных шести формах отражают общий механизм существования не просто метода учебно-профессионального развития, но выражают генезис развития смысла генеральной линии экспозиции развития целостно-системной широкопрофильной личности, спроектированной педагогометрическими функциями математического моделирования при реализации международных образовательных стандартов морского образования эревнометрической формы алигорамного содержания.

\section{References:}

1. Mishchik SA (2014) Pedagogometrika and mathematical modeling educational activity. Materialy Mezhdunarodnoy nauchnoy konferenctsii "Modern mathematics in science"
- 30.06.2014. ISJ Theoretical \&Applied Science 6(14): 54-56 Caracas, Venezuela. doi: http://dx.doi.org/10.15863/TAS.2014.06.14.10 


\begin{tabular}{|c|c|c|c|c|c|c|}
\hline Impact Factor: & $\begin{array}{l}\text { ISRA (India) } \\
\text { ISI (Dubai, UAF } \\
\text { GIF (Australia) } \\
\text { JIF }\end{array}$ & $\begin{array}{l}=1.344 \\
=0.829 \\
=0.564 \\
=1.500\end{array}$ & $\begin{array}{l}\text { SIS (USA) } \\
\text { PИНЦ (Russia) } \\
\text { ESJI (KZ) } \\
\text { SJIF (Morocco) }\end{array}$ & $\begin{array}{l}=0.912 \\
=0.207 \\
=\mathbf{3 . 8 6 0} \\
=\mathbf{2 . 0 3 1}\end{array}$ & $\begin{array}{l}\text { ICV (Poland) } \\
\text { PIF (India) } \\
\text { IBI (India) }\end{array}$ & $\begin{array}{l}=6.630 \\
=1.940 \\
=4.260\end{array}$ \\
\hline
\end{tabular}

2. Mishchik SA (2014) Simulation training activity methods of mathematical logic. Materialy Mezhdunarodnoy nauchnoy konferenctsii "Eurapean Science and Education" - 30.07.2014. ISJ Theoretical \&Applied Science 6(15): 72-74 Marseille, France. doi: http://dx.doi.org/10.15863/TAS.2014.07.15.13

3. Mishchik SA (2014) Mathematical modeling system integrity-cycle of life activity - first goal pedagogometriki. Materialy Mezhdunarodnoy nauchnoy konferenctsii "European Applied Sciences" - 30.08.2014. ISJ Theoretical \&Applied Science 7(16): 77-79. Aix-en-Provence, France. doi: http://dx.doi.org/10.15863/TAS.2014.08.16.13

4. Mishchik SA (2014) Mathematical modeling system integrity-curricular activities - the second problem pedagogometriki. Materialy Mezhdunarodnoy nauchnoy konferenctsii "European Innovation" - 30.09.2014. ISJ Theoretical \&Applied Science 9(17): 126-128 Martigues, France. doi: http://dx.doi.org/10.15863/TAS.2014.09.17.21

5. Mishchik SA (2014) Mathematical modeling holistic-systemic communicative activity - the third task pedagogometriki. Materialy Mezhdunarodnoy nauchnoy konferenctsii "European Scientific Achievements" 30.10.2014. ISJ Theoretical \&Applied Science 10(18): 45-47 Brighton, UK. doi: http://dx.doi.org/10.15863/TAS.2014.10.18.11

6. Mishchik SA (2014) Mathematical modeling integrity - system performance subject - fourth task pedagogometriki. Materialy Mezhdunarodnoy nauchnoy konferenctsii "Eurapean Science and Technology" 30.11.2014. ISJ Theoretical \&Applied Science 11(19): 51-54 Southampton, UK. doi: http://dx.doi.org/10.15863/TAS.2014.11.19.10

7. Mishchik SA (2015) Pedagogometrik - science and academic subject. Materialy Mezhdunarodnoy nauchnoy konferenctsii "European Technology in Science" 28.02.2015. ISJ Theoretical \& Applied Science 02 (22): 103-106 Malmö, Sweden. doi: http://dx.doi.org/10.15863/TAS.2015.02.22.17

8. Irodov I. E. (1979) Zadachi po obshhej fizike. M.: Nauka, 1979 - p.368.
9. Tokmazov GV (2014) Matematicheskoe modelirovanie $\mathrm{v}$ uchebno-professional'noy deyatel'nosti. Materialy Mezhdunarodnoy nauchnoy konferentsii «Modern mathematics in science» - 30.06.2014. ISJ Theoretical \& Applied Science 6(14): 44-46. - Caracas, Venezuela. doi: http://dx.doi.org/10.15863/TAS.2014.06.14.8

10. Tokmazov GV (2014) Mathematical modeling research skills in educational activity methods of probability theory. Materialy Mezhdunarodnoy nauchnoy konferenctsii "Eurapean Science and Technology" 30.11.2014. ISJ Theoretical \&Applied Science 11(20): 66-69 Southampton, United Kingdom. doi:

http://dx.doi.org/10.15863/TAS.2014.11.19.13

11. Mishhik N.A. (2016) Pravovy'e osnovy' francuzskoj si-stemy' bor'by' s zagryazneniem morya / Nauchny'e issledovaniya: Informaciya, analiz, prognoz [Tekst]: monografiya / [V.E'.Lebedev, A.A.Sviridenko, V.M.Sokolinskij i dr.]; pod obshhej red. prof. O.I.Kirikova - Kniga 51.- Voronezh-Moskva, 2016.

12. Mishchik NA (2014) The practice of french justice article 228 of the UN convention on the law of the sea. Materialy Mezhdunarodnoy nauchnoy konferenctsii "The European Science and Education"- 30.07.2014. ISJ Theoretical \& Applied Science 07 (15): 93-97. - Marseille, France.

doi: http://dx.doi.org/10.15863/TAS.2014.07.15.19

13. Mishhik N.A., Antonenko G.A. (2013) Liniya gorizonta kak gradientny'j perepad $\mathrm{V}$ fotograficheskix izmereniyax dlya celej morexodnoj astronomii//E'kspluataciya morskogo transporta. 2013. № 2 (72). Novorossijsk, p. 23-28.

14. Mishhik N.A. (2000) Optimizaciya metodov morexodnoj astronomii [Tekst]: avto-ref.dis. ... kand. tex. nauk: 05.22.16 / N.A.Mishhik. Novorossijsk, 2000. - 24 p.

15. Mishhik N.A. (2000) Optimizaciya metodov morexodnoj astronomii [Tekst]: dis. ... kand. tex. nauk: 05.22.16 / N.A.Mishhik. Novorossijsk, 2000. - 188 p. 